\title{
Oketani Lactation Management: A New Method to Augment Breast Milk
}

\author{
N KABIR, S TASNIM
}

(J Bangladesh Coll Phys Surg 2009; 27: 155-159)

\begin{abstract}
Introduction
The beneficial effect of breastfeeding both for mother and baby are well known. Though breastfeeding culture is present in Bangladesh, mothers easily deviate from nursing with breastmilk when they face some confusion or trouble. Most mothers do not know about her capacity to feed her child and it is more than the need of her child. They are to be prepared for breastfeeding from antenatal period, have to be given support during initiation of breastfeeding and in the postnatal period. Counseling and correcting position and attachment are recognized as management skill but 33-37\% mothers further needed Oketani Lactation Management for establishment of successful breastfeeding respectively. 1,2
\end{abstract}

The Oketani Lactation Management is a unique breast management created by Ms. Sotomi Oketani ${ }^{3}$ of Japan. Sotomi also theorized that breast feeding enhances mother and child bonding while also encircling the mother and child's physical and mental condition by natural means. It is widely practiced in Japan for several years. It helps lactating mothers to overcome certain difficulties while breastfeeding their babies. It was first introduced in Bangladesh in 1994. It is also known as Oketani breast treatment.

In order to enable an infant to nurse successfully "who is the best physician for the breast" it is necessary to constantly keep the breast and nipple in good condition. Babies can suckle the breast easily with the improvement of the elasticity of the nipple and the areola. The Oketani technique or breast

Dr. Nazneen Kabir, Dr. Saria Tasnim, Associate Professor of OBGYN, Institute of Child and Mother Health (ICMH), Dhaka

Address of Correspondence to: Dr. Nazneen Kabir, Associate Professor of OBGYN, Institute of Child and Mother Health (ICMH), Dhaka

Received: 25 February, 2009

Accepted: 10 July, 2009 management enables the baby to achieve this and keep the breast in the best condition.

History of Lactation Management

'Oketani Lactation Management' was formalized in 1981 in Japan. Hirst states that massages performed during the first part of the puerperal period can prevent the breastmilk clogging in the breast allowing free flow of breastmilk. De Lee ${ }^{4}$ also states that effective results were achieved when different massages were used respectively for breastmilk insufficiency and clogged breasts. M Ebner 5 supports the use of exercises to stimulate the greater pectoral muscle to increase the production of breast milk and the use of hot and cold compression and massage to increase the flow of blood to the mammary glands as an alternative method. In Japan, breast massage has been used in eastern medicine since ancient times. In 1985 "Shufu no Tomo" Breastfeeding Counseling Service specializes in the Oketani Method of Breast Care ${ }^{6}$.

\section{Anatomy of the breast ${ }^{7}$}

The breast is divided into different sections for applying Oketani technique. The areas differ for right and left breast. First a perpendicular line is drawn from the nipple towards the breast line. Using these as the baseline, and an area of $105^{\circ}$ is measured on both sides and named $\mathrm{B}$ and $\mathrm{C}$. A stands for the remaining $150^{\circ}$ on the top of both breasts, B stands for the inner sides of the right breast and outer sides of the left breast, while $\mathrm{C}$ stands for the outer side of the right breast and inner side of the left breast. Both B and C are $105^{\circ}$ on each side.

Then each of the A, B and $\mathrm{C}$ sections are subdivided into three more parts. In both left and right breasts, the section $\mathrm{A}$ is divided into three equal parts 1,2, and 3 starting clock wise, while the sections $\mathrm{B}$ and $\mathrm{C}$ are equally divided from the top to the bottom (1), (2) and (3). Namely, B-(3) and C-(3) are along side each other 


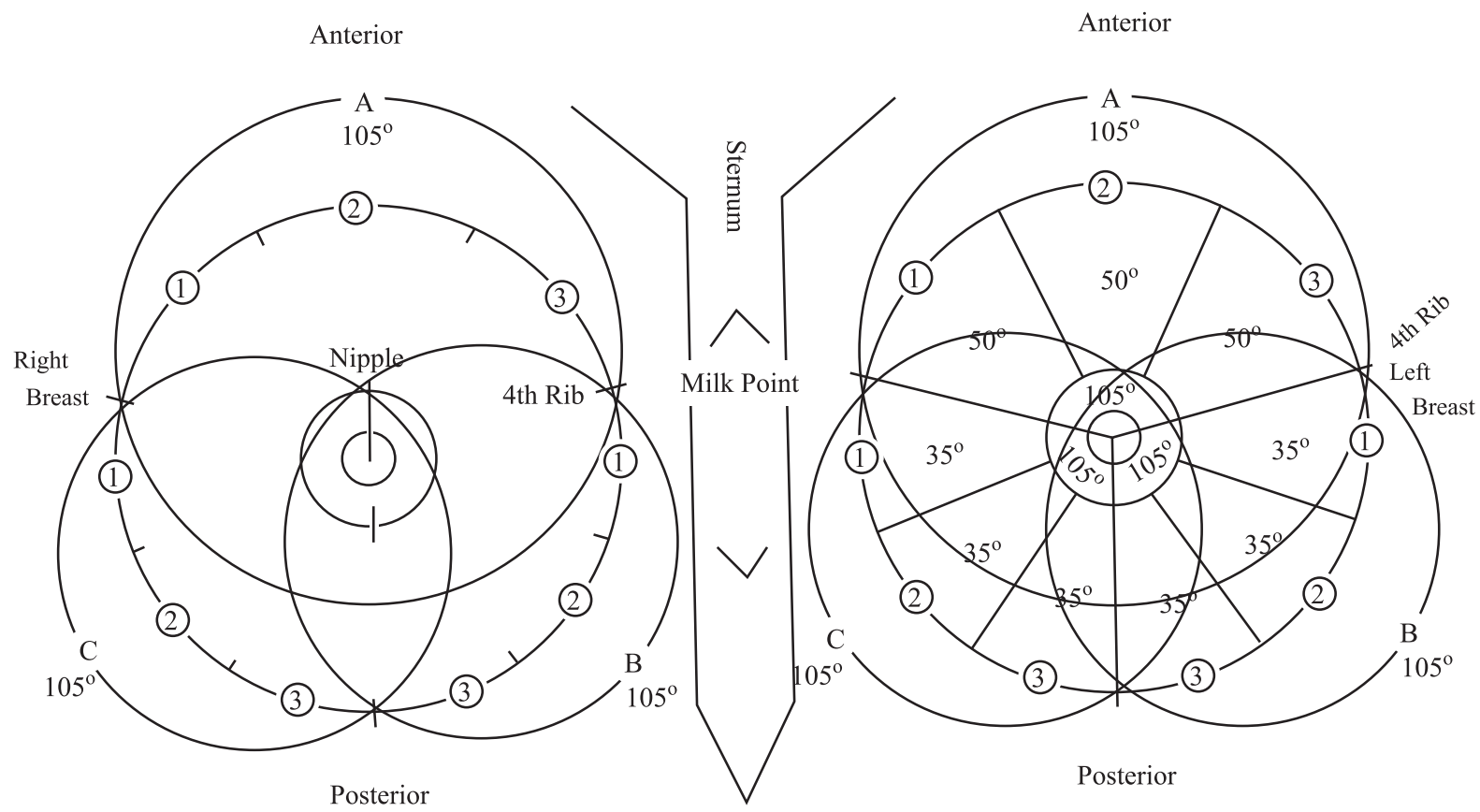

Fig.: Anatomy of the breasts in relation to Oketani

and define the boundary of $\mathrm{B}$ and $\mathrm{C}$ in the centre. $\mathrm{B}$ (3) and C-(3) are on the axis of the breast which support it when standing ${ }^{3}$.

\section{Basics of Oketani massage}

Breast is composed of the mammary glands which are surrounded by skin, connective tissue and the adipose tissue. Posteriorly, the mammary glands are loosely connected to the deep fascia of the pectoralis major. The breast is movable against the pectoralis major muscle and the thorax. The location of the breast is fixed by the connective tissue to the skin and pectoral muscle. This supporting connective tissue possess elasticity and spontaneously expands and contracts to accommodate physiological function of the breast.

The deep fascia acts as the base of the breasts. If the base loses its elasticity due to any cause, it appears to adhere to the pectoralis major fascia. If milk is not excreted under such condition the pressure in the breast rises, the circulation of venous blood is impaired and the mammary veins becomes congested. At the same time areola and nipple becomes indurated.

Oketani manual technique dissolve the disorder by manual separation of adhesions between the breast base and pectorals major fascia helping to restore the normal breast function. It also makes the breast more elastic and soft.

This operation is called the opening of the mammary depths. Basic mechanism of breast is push ups and pull ups. The idea is to mobilize breast from its base to increase its vascularity and thereby increase milk flow ${ }^{7}$.

\section{Characteristics $^{8}$}

1) Oketani's breast manipulation gives no discomfort or pain to the patient

2) The patient will suddenly feel general relief and comfort

3) Lactation is enhanced regardless to the size or shape of the patient's breasts and nipples

4) The quality of milk is improved

5) Deformity such as inversion, flattening or cracking of the nipple is rectified

6) Nipple injuries and mastitis are prevented

\section{How does the technique work?}

In order to separate the adhesions, breast is to be pushed forward manually by inserting a hooked finger into the basal portion to a depth of about 0.8 $\mathrm{cm}$ lifting the breasts forward. After that the adhesions is gently pushed back towards the pectoral muscle depending on the reactions of the breastfed mother. As a result, the operator feels a gap between 
breast base and the pectoralis major. When the breast adhesions are loosened the entire breast shows better elasticity and this results in softer, more pliable nipples with improved lactiferous ducts contour and suckling. Short rhythmical pull up separate the basal portion of the breast from the pectoral muscle by distance of 1-2 cm. As a result, the breast outline becomes more distinct.

\section{Procedures}

There are 8 different techniques in the manual operation of Oketani Breast Treatment. They are numbered (1), (2), (3), (4), (5), (6), (7) and (8) expressing or milking. Operations (1) to (7) are called one set or course of treatment. One set of operations and expressing are completed within one minute and this is repeated for $15-20$ minutes.

\section{Manipulation 1}

Pushing up C (1) and pulling up A (1), B (2) by third finger of right hand and little finger of left hand toward left shoulder.

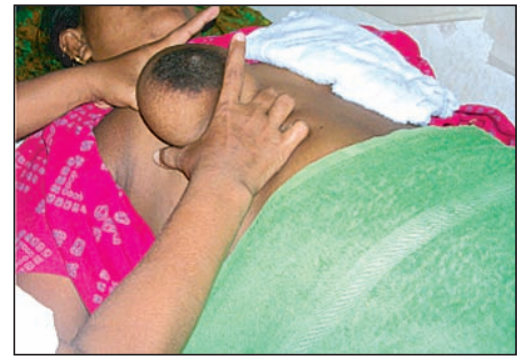

Manipulation 1

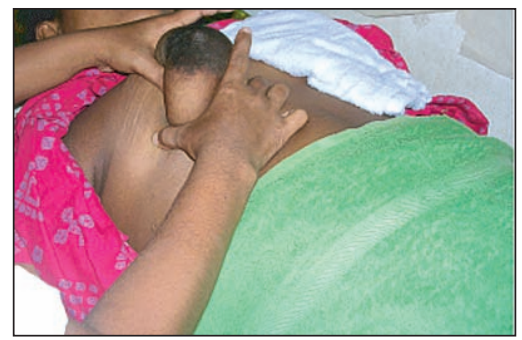

Manipulation 2

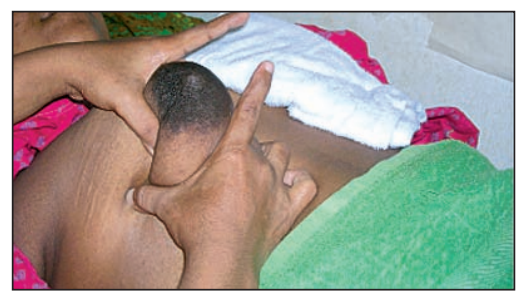

Manipulation 3

\section{Manipulation 2}

Pushing up C (1-2) and pulling up of central portion of $A(1-2)$ and $B(1-2)$ by third finger of both hand toward left axilla.

\section{Manipulation 3}

Pushing up C (2) and pulling up A (3) and B (1) by third finger and thumb of right hand and third finger of left hand placing the thumb above the second joint of the right thumb. Here pushing and pulling will be parallel to opposite breast.

Pushing and pulling numbered (1), (2) and (3) are the manipulations used to separate hard portion of the breast from the fascia of the pectoralis major.

\section{Manipulation 4}

Pushing down the whole breast towards the umbilicus placing the right thumb on $\mathrm{C}(1)$, middle, third, and little finger on side B and left thumb on $\mathrm{C}$ (1), middle, third, and little finger on side A.

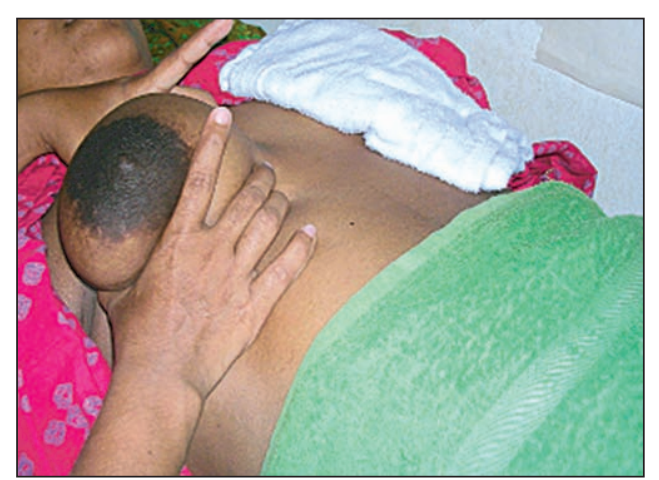

Manipulation 4

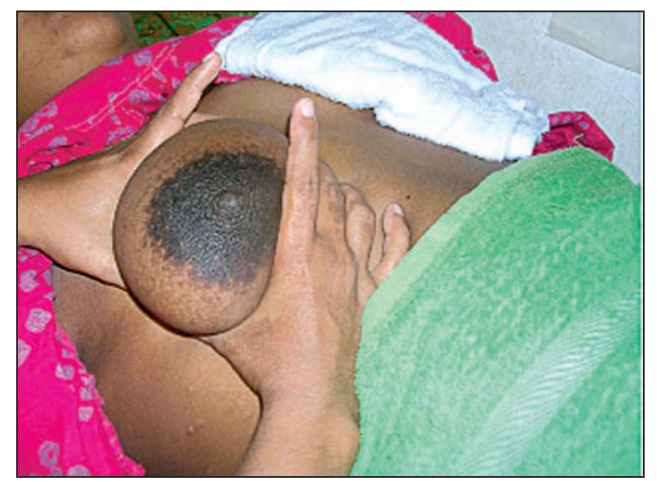

Manipulation 5 


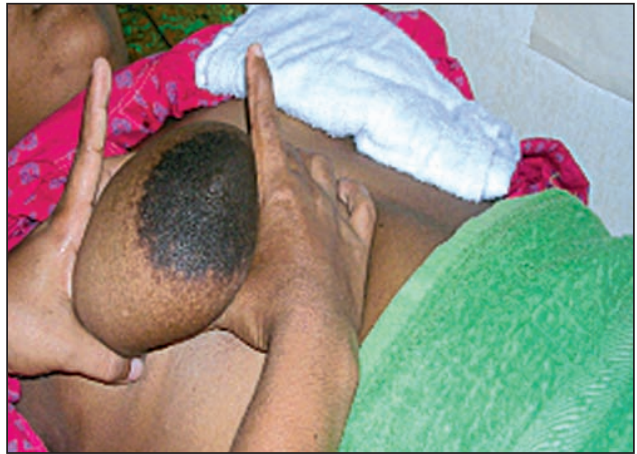

Manipulation 6

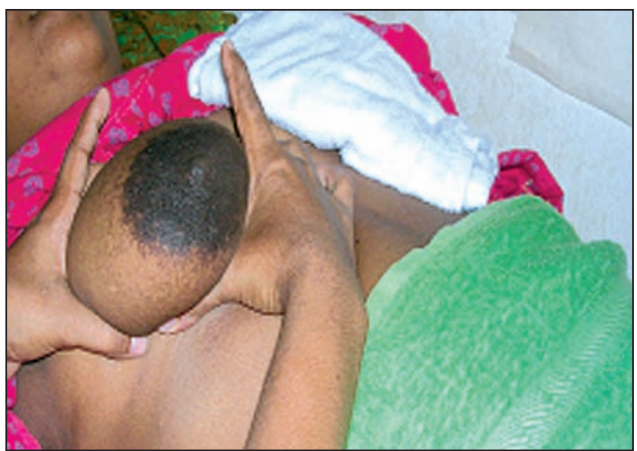

Manipulation 7

\section{Manipulation 5}

Pulling down the breast towards the direction of the practitioner by the right hand while gently twisting it from the upper periphery to the lower margin holding the breast like manipulation 4

\section{Manipulation 6}

Pulling down the breast towards the direction of the practitioner by the left hand while gently twisting it from the upper periphery to the lower margin holding the breast like manipulation 5 . It is a procedure opposite to operation (5).

Manual procedures (5) and (6) are techniques to isolate the hard base portions of the breast $\mathrm{C}-(2)$ to $\mathrm{C}$ (1) from the pectoralis major fascia.

\section{Manipulation 7}

Slowly rotate the breast in a clockwise direction and note the elasticity of the base of the breast

\section{Manipulation $8(A-D)$}

Expression is done in four different directions for outside surface (8A), lower part (8B), inside of the breast $(8 \mathrm{C})$ and inside of the upper periphery of the right breast (8D) and inside, lower part, outside surface and inside of the upper periphery of the left breast.
Manual operations are performed rhythmically. Operations with no rhythm may give an uncomfortable feeling to the patient.

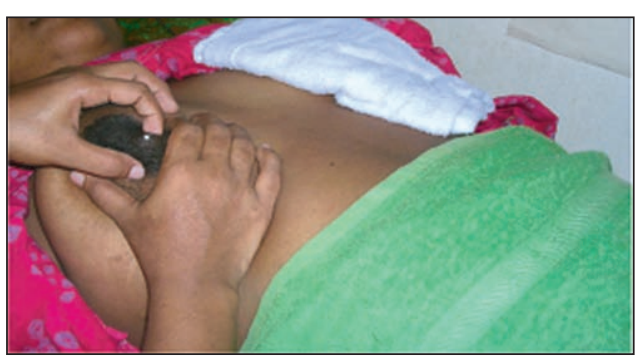

Manipulation- $8 A$

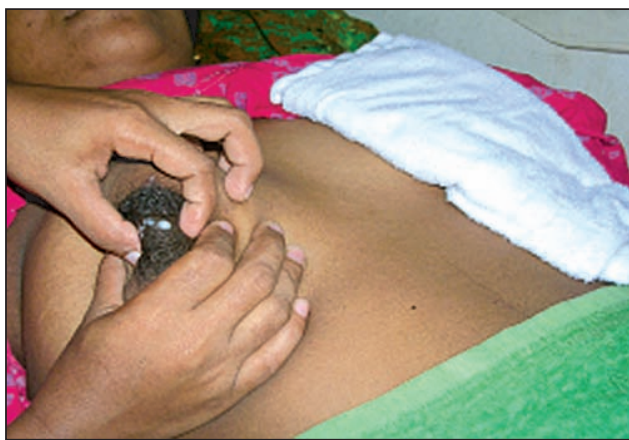

Manipulation- $8 B$

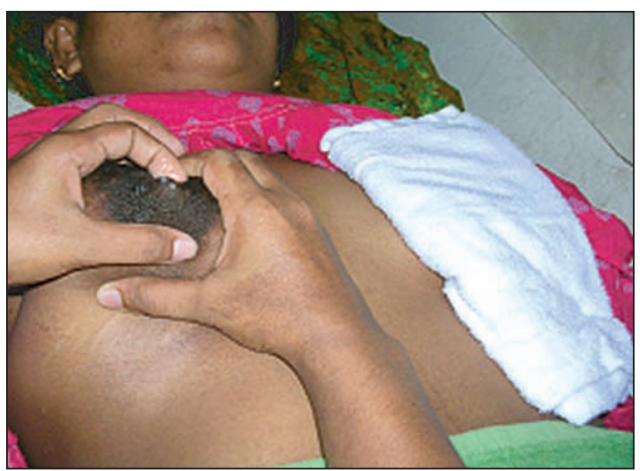

Manipulation- $8 C$

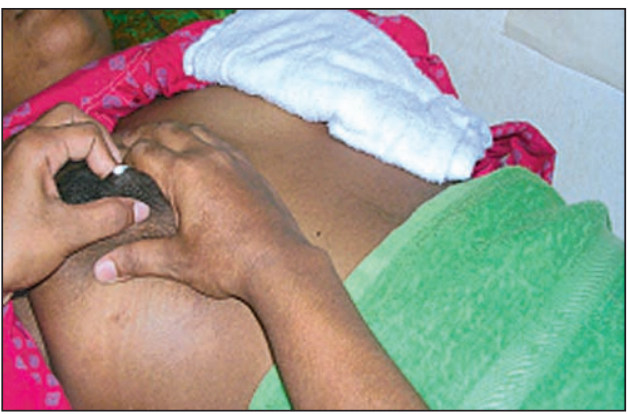

Manipulation- $8 D$

Manipulation $8(A-D)$ 


\section{Discussion}

Breastfeeding is the best method of nursing newborn babies and the rich quality of breast milk as a source of nourishment is known throughout the world. It is well established that proper positioning and attachment is most crucial for effective breastfeeding. After correcting the position and attachment some of the mothers require further help for improving lactation. It is reported that Oketani breast massage stimulate the physical and mental development of infants improving both their mood and sleep pattern by raising the quality of excreted milk ${ }^{9}$. Oketani manual technique of breast management result in softening of the entire breast enhancing the pliability of areola that becomes elastic and pink, lactiferous ducts and nipples that also become more elastic and rounded by separating adhesion from underlying breast base. The whole breast become more supple produce good quality breast milk due to increased total solids, lipids concentration and gross energy 10 . Mervat I showed that whey protein concentration was increased and casein was decreased by breast massage ${ }^{11}$. Milk lipids in the pre massage samples increased from one day to nine months and then decreased slightly after nine months ${ }^{12,13}$. The increase of lipid and total solid change the taste of the breast milk and become less greasy, yellow and sweet. Due to increased energy supply from lipid babies hunger is more easily satisfied. Ohno demonstrated that release of opioid, small peptides and decrease of lipooxygenase activity by the Oketani Massage may improve infant development ${ }^{14}$.

Oketani breast massage also is used as management skill for breastfeeding problems, breast problems like not enough milk, low milk production, partial breastfeeding and engorgement of breast. Repeated manual operation normalise the opening of lactiferous duct and the baby find it much easier to suckle and continue to suckle efficiently. It also improve breast circulatory system which cause excretion of better quality milk. The mothers feel comfort and refreshed all over the body after loosening the deep breast tissues by manual technique like suddenly being allowed to breath.

When the mother and baby is treated by Oketani technique for augmentation of breast milk, it provides great satisfaction and enthusiasm for both of them and as a consequence the growth and development of the baby is ensured. In order to produce good quality milk, breast massage by Oketani certified midwives is recommended.

\section{References}

1. Kabir $\mathrm{N}$ et al. Activities of Lactation Management Centre (LMC) at a National level Hospital in Bangladesh. Bangladesh J Child Health 2004; 28: 76-80

2. Oketani S. The Real Report of the Oketani's Manual technique of Breast Treatment - The Practice. 1992; 14

3. Manual of Oketani Breast Massage Technique, Akaeda Medical Research Foundation, Tokyo, Japan, 1996.

4. [No authors listed]. The use and assistance given by the "Shufu no Tomo" Breast Feeding Counseling Service. Josanpu Zassi. 1987 Mar,41(3):230-8.

5. Michiko Kobayashi, Masayo Awano, Saeko Nagao. Oketani Lactation Management. Japanese Breast Massage Manual. Oketani Lactation Management Association. Japan, 2004

6. Kobayashi M. Promoting breast-feeding. A successful regional project in Japan. Acta Paediatr Jpn 1989;31:403-10.

7. Foda, Mervat I, Kawashima, Takaaki, Nakamura, Sadako, Kobayashi, Michiko, Oku, Tsuneyuki. Composition of Milk Obtained From Unmassaged Versus Massaged Breasts of Lactating Mothers. Journal of Pediatric Gastroenterology and Nutrition 2004;38(5):484-487.

8. Nommsen LA, Lovelady CA, Heining MJ. Determinants of energy, protein, lipid and lactose concentrations in human milk during the first 12 months of lactation: the DARLING Study. Am J Clin Nutr 1991;53:457-65.

9. Jensen GR, Bitman J, Carlson SE, et al. Human milk lipids. In Jensen GR, ed. Handbook of Milk Composition (5th ed): Academic Press; 1995.

10. Ohno N. Change of composition and physiological activity in milk obtained by the Oketani breast massage method [PhD thesis]. Nagano, Japan: Shinshu University, Department of Agriculture; 2001. 